\title{
The Dirac Propagator for One-Dimensional Finite Square Well
}

\author{
P. Kongkhuntod, N. Yongram \\ Department of Physics, Faculty of Science, Naresuan University, Phitsanulok, Thailand \\ Email: phonphimonk61@nu.ac.th, nattapongy@nu.ac.th
}

How to cite this paper: Kongkhuntod, P. and Yongram, N. (2020) The Dirac Propagator for One-Dimensional Finite Square Well. Journal of Modern Physics, 11, 1639-1648.

https://doi.org/10.4236/jmp.2020.1110102

Received: August 28, 2020

Accepted: October 23, 2020

Published: October 26, 2020

Copyright (c) 2020 by author(s) and Scientific Research Publishing Inc.

This work is licensed under the Creative Commons Attribution International License (CC BY 4.0).

http://creativecommons.org/licenses/by/4.0/

\section{Open Access}

\begin{abstract}
The solution of Dirac particles confined in a one-dimensional finite square well potential is solved by using the path-integral formalism for Dirac equation. The propagator of the Dirac equation in case of the bounded Dirac particles is obtained by evaluating an appropriate path integral, directly constructed from the Dirac equation. The limit of integration techniques for evaluating path integral is only valid for the piecewise constant potential. Finally, the Dirac propagator is expressed in terms of standard special functions.
\end{abstract}

\section{Keywords}

Path-Integral, Dirac Equation, Dirac Propagator, Finite Square Well

\section{Introduction}

The solution of quantum mechanical problems in non-relativistic Schrödinger and relativistic Dirac equation with a finite square well potential which were conceptually very relevant for our understanding about 80 years ago seem to be no longer of primary importance. Occasionally, however, there is a new line of inquiry into this problem typically by applying new mathematical technique [1-5]. Moreover the finite square well potential is of great practical importance since it forms the basis for understanding low-dimensional structures such as quantum well devices.

For relativistic quantum mechanics, the problem of a Dirac particle confined in a finite square well potential is a useful tool to discuss, in advanced quantum mechanics courses. The issues arise when one extends quantum mechanics to incorporate special relativity. In a series of papers $[6,7]$ titled "The Relativistic One-Dimensional Square Potential" and the textbooks of Relativistic Quantum Mechanics [8] the problem of relativistic spin- $\frac{1}{2}$ particle (or Dirac particle) confined in a finite square well potential is studied by solving the one-dimensional Dirac equation. It is surprising that this problem has not been solved 
using path integral approach despite the fact that one can do so for the non-relativistic counterpart, for example Nevels et al. [9] evaluated the path integral when a spinless particle encounters an infinite potential barrier, Janke and Kleinert [10] exhibited the path integral for particle in a box (infinite square well), Goodman [11] solved the problem of the infinite squarewell potential in one dimension using the path integral, and Barut and Duru [12] evaluated the exact path integral for the propagator for square potential barriers in one dimension and for the radial square-well potential in two dimensions. Here we will solve the problem of Dirac particle confined in a finite square well potential via the path integral for Dirac equation.

It is fair to say that the Dirac equation and its propagator are more fundamental concepts than the Schrödinger equation and its propagator. There have been attempts to write down a path integral expression for the Dirac propagator similar to the Feynman path integral expression for the Schrödinger propagator. For example Riazanov [13] showed that the Feynman path integral is identical to the propagation function of the Dirac equation. Papadopoulos and J. T. Devreese [14] obtained the Dirac propagator through the Feynman path integral [15-18], directly constructed from the Dirac equation. Gaveau and Schulman [19] constructed path integral formula for the Dirac propagator in three spatial dimensions, called the "projector path" summation, which is one of a generalization of the Feynman "checkerboard" propagator [18]. Rosen [20] discovered that the Feynman path summation for the one-dimensional Dirac equation can be projected into three spatial dimensions to yield a path-summation formula for physical spin- $\frac{1}{2}$ particles of nonzero 2 mass. Since the threespace projection matrix is independent of time and does not involve the particle's mass, relativistic motion governed by the Dirac equation has an underlying one-dimensional aspect.

The purpose of the present paper is to write down the Dirac propagator for Dirac particles confined in a one-dimensional square well potential of depth $V_{0} \leq 0$ and width $a$. The organization of the rest of the paper is as follows: In Section 2 we evaluate the propagator of the Dirac particle in a square well. Finally, we summarize our results in Section 3.

\section{Dirac Propagator for a One-Dimensional Square Well}

This section deals with the construction and the evaluation of the propagator of Dirac particles confined in an one-dimensional finite square well potential of depth $V_{0} \leq 0$ and width $a$. The onedimensional Dirac Hamiltonian for the motion in one dimension under the influence of square well potential is

$$
H=H_{0}+V(x)
$$

where

$$
H_{0}=-\mathrm{i} c \hbar \alpha_{x} \frac{\partial}{\partial x}+m_{0} c^{2} \beta
$$

is the free particle Hamiltonian, the matrix $\alpha_{x}$ is

$$
\alpha_{x}=\left(\begin{array}{cc}
0 & \sigma_{x} \\
\sigma_{x} & 0
\end{array}\right)
$$


where the Pauli matrix $\sigma_{x}=\left(\begin{array}{ll}0 & 1 \\ 1 & 0\end{array}\right)$, the matrix $\beta$ is

$$
\beta=\left(\begin{array}{cc}
\mathbf{1} & 0 \\
0 & -1
\end{array}\right)
$$

where $\mathbf{1}=\left(\begin{array}{ll}1 & 0 \\ 0 & 1\end{array}\right)$ is identity $2 \times 2$ matrix, and $V(x)$ denotes square well potential

$$
V(x)= \begin{cases}-V_{0}, & \text { for }-a / 2<x<a / 2 \\ 0 & \text { for } x \leq-a / 2, x \geq a / 2\end{cases}
$$

The propagator $K\left(x_{b}, t_{b} ; x_{a}, t_{a}\right)$ satisfies the Dirac equation

$$
\left(\mathrm{i} \hbar \frac{\partial}{\partial t}-H\right) K=0
$$

The short-time propagator of the above equation is given by

$$
K\left(x_{j}, t_{j-1}+\varepsilon ; x_{j-1}, t_{j-1}\right)=\exp \left(-\frac{\mathrm{i}}{\hbar} H \varepsilon\right) I_{4} \delta\left(x_{j}-x_{j-1}\right)
$$

where $I_{4}$ is the $4 \times 4$ identity matrix.

The finite-time propagator can be constructed via the composition law

$$
\begin{aligned}
K\left(x_{b}, t_{b} ; x_{a}, t_{a}\right)= & \lim _{\varepsilon \rightarrow 0}\left[\int_{-a / 2}^{a / 2} \prod_{j=1}^{N-1} \mathrm{~d} x_{j} \prod_{j=1}^{N} K\left(x_{j}, t_{j-1}+\varepsilon ; x_{j-1}, t_{j-1}\right)\right. \\
& +\int_{-\infty}^{-a / 2} \prod_{j=1}^{N-1} \mathrm{~d} x_{j} \prod_{j=1}^{N} K_{0}\left(x_{j}, t_{j-1}+\varepsilon ; x_{j-1}, t_{j-1}\right) \\
& \left.+\int_{a / 2}^{\infty} \prod_{j=1}^{N-1} \mathrm{~d} x_{j} \prod_{j=1}^{N} K_{0}\left(x_{j}, t_{j-1}+\varepsilon ; x_{j-1}, t_{j-1}\right)\right]
\end{aligned}
$$

This is the propagator for Dirac particles confined in an onedimensional finite square well, then, each integral is limited to the domain $-a / 2<x<a / 2$, otherwise the propagators for free Dirac particles are taken into account, and allows all imaginable paths, including those with velocities greater than that of light. It seems like the principles of relativistic mechanics is being violated. However, the situation is similar to that of nonrelativistic quantum mechanics in which the non-classical paths are allowed.

The first term on right-hand side (RHS) of (8) depends only on the short-time propagator

$$
\begin{aligned}
K\left(x_{j}, t_{j-1}+\varepsilon ; x_{j-1}, t_{j-1}\right) & =x_{j}\left|\exp \left(-\frac{\mathrm{i}}{\hbar} \varepsilon H\right)\right| x_{j-1} \\
& =x_{j}\left|\exp \left(-\frac{\mathrm{i}}{\hbar} \varepsilon H_{0}-\frac{\mathrm{i}}{\hbar} \varepsilon V\left(x_{j}\right)\right)\right| x_{j-1}
\end{aligned}
$$


It can be approximate up to the order of $O\left(\varepsilon^{2}\right)$ using Zassenhaus formula [21-24]. Equation (9) becomes

$$
\begin{aligned}
K\left(x_{j}, t_{j-1}+\varepsilon ; x_{j-1}, t_{j-1}\right) & =x_{j}\left|\mathrm{e}^{-\frac{\mathrm{i}}{\hbar} \varepsilon H_{0}}\right| x_{j-1} \mathrm{e}^{-\frac{\mathrm{i}}{\hbar} \varepsilon V\left(x_{j}\right)} \\
& =K_{0}\left(x_{j}, t_{j-1}+\varepsilon ; x_{j-1}, t_{j-1}\right) \mathrm{e}^{-\frac{\mathrm{i}}{\hbar} \varepsilon V\left(x_{j}\right)}
\end{aligned}
$$

It is important to observe the order of the various $K_{0}\left(x_{j}, t_{j-1}+\right.$ $\left.\varepsilon ; x_{j-1}, t_{j-1}\right)$ in (10); they are noncommuting matrices and their time ordering relates to the way a given spinor evolves according to the Dirac dynamics.

The Dirae equation, being of first order in time, enables one to write a short-time free propagator $K_{0}\left(x_{j}, t_{j-1}+\varepsilon ; x_{j-1}, t_{j-1}\right)$ in terms of the infinitesimal generator $\left[I_{4}-(\mathrm{i} / \hbar) \varepsilon H_{0}\right]$ as

$$
K_{0}\left(x_{j}, t_{j-1}+\varepsilon ; x_{j-1}, t_{j-1}\right)=\left(I_{4}-\frac{\mathrm{i}}{\hbar} \varepsilon H_{0}\right) \delta\left(x_{j}-x_{j-1}\right)
$$

The expression (11) can also be obtained by expanding the exact expression for the propagator to the first order in time.

By operating $I_{4}-\frac{i}{\hbar} \varepsilon H_{0}$ on the Fourier representations of $\delta$ functions, the short-time free propagator becomes

$$
\begin{aligned}
& K_{0}\left(x_{j}, t_{j-1}+\varepsilon ; x_{j-1}, t_{j-1}\right) \\
& =\int\left[I_{4}-\frac{\mathrm{i}}{\hbar} \varepsilon\left(c \hbar \alpha_{x} k_{j}+m_{0} c^{2} \beta\right)\right] \times \exp \left[\mathrm{i} k_{j}\left(x_{j}-x_{j-1}\right)\right] \frac{\mathrm{d} k_{j}}{2 \pi}
\end{aligned}
$$

Here we also use the fact that $H_{0}$ does not operate on $V\left(x_{j}\right)$ because it is a constant potential.

Inserting (10) and (12) into (8) and making use of isomeric time partitions, i.e., all $\varepsilon$ of the $N$ th partition will be taken equal to $\varepsilon / N$, we obtain for the finite-time propagator the expression

$$
\begin{aligned}
& K\left(x_{b}, t_{b} ; x_{a}, t_{a}\right) \\
& =\lim _{N \rightarrow \infty} \frac{1}{(2 \pi)^{N}}\left[\int_{-a / 2}^{a / 2} \prod_{j=1}^{N-1} \mathrm{~d} x_{j} \prod_{j=1}^{N} \mathrm{~d} k_{j} \kappa_{j} \exp \left(-\frac{\mathrm{i}}{\hbar} \frac{\varepsilon V\left(x_{j}\right)}{N}\right)\right. \\
& \left.+\int_{-\infty}^{-a / 2} \prod_{j=1}^{N-1} \mathrm{~d} x_{j} \prod_{j=1}^{N} \mathrm{~d} k_{j} \kappa_{j}+\int_{a / 2}^{\infty} \prod_{j=1}^{N-1} \mathrm{~d} x_{j} \prod_{j=1}^{N} \mathrm{~d} k_{j} \kappa_{j}\right] \mathrm{e}^{\mathrm{i} k_{j}\left(x_{j}-x_{j-1}\right)}
\end{aligned}
$$

where $\kappa_{j}$ is a $4 \times 4$ matrix given by

$$
\kappa_{j}=I_{4}-\frac{\mathrm{i}}{\hbar} \frac{\varepsilon}{N}\left(c \hbar \alpha_{x} k_{j}+m_{0} c^{2} \beta\right)
$$

Again it should be noted that as far as (13) is concerned the order of the $\kappa_{j}$ matrices is important. Equation (13) is already a form of the path integral giving the propagator of the Dirac particle under the influence of a potential $V(x)$. The summation over all paths starting from $x_{a}$ at time $t_{a}$ and ending at position $x_{b}$ at time $t_{a}$ is attained through the infinitely multiple process of integration. 
The integrations over various $k_{j}$ 's are essentially path summations in momentum space. However, one would like to express the summation in term of paths in configuration space. This is easily done in the following manner: the short-time propagators entering the process of multiple integration need only be taken to the first order in $\varepsilon / N$. If we replace $-\mathrm{i} c k_{j} \varepsilon / N$ in $(13)$ by $\left[\exp \left(-\mathrm{i} c k_{j} \varepsilon / N\right)-1\right]$ and put the resulting $\kappa_{j}$ in (12), the limit as $N \rightarrow \infty$ will not be affected. With the above replacement we are able to perform the integrations, and the resulting path integral in terms of configuration paths alone is given by

$$
\begin{aligned}
& K\left(x_{b}, t_{b} ; x_{a}, t_{a}\right) \\
& =\lim _{N \rightarrow \infty}\left[\int_{-a / 2}^{a / 2} \prod_{j=1}^{N-1} \mathrm{~d} x_{j} \exp \left[-\frac{\mathrm{i}}{\hbar} \frac{\varepsilon}{N} V\left(x_{j}\right)\right]+\int_{-\infty}^{-a / 2} \prod_{j=1}^{N-1} \mathrm{~d} x_{j}\right. \\
& \left.+\int_{a / 2}^{\infty} \prod_{j=1}^{N-1} \mathrm{~d} x_{j}\right]\left\{\left[I_{4}-\alpha_{x}-\frac{\mathrm{i}}{\hbar} \frac{\varepsilon}{N} m_{0} c^{2} \beta\right] \delta\left(x_{j}-x_{j-1}\right)\right. \\
& \left.+\alpha_{x} \delta\left(x_{j}-x_{j-1}-c \frac{\varepsilon}{N}\right)\right\}
\end{aligned}
$$

This propagator is valid for piecewise constant potential. The product of the various short-time propagators in (15) is ordered from right to left in increasing order of time.

We found that the difficulties in evaluating the integrand (15) are now obvious; the Gaussian integrals cannot be evaluated in closed form if them are restricted to a bounded domain. We repeat the integration of (8) with additional following term

$$
\begin{aligned}
& \int_{-a / 2}^{a / 2} \prod_{j=1}^{N-1} \mathrm{~d} x_{j} \prod_{j=1}^{N} K_{0}\left(x_{j}, t_{j-1}+\varepsilon ; x_{j-1}, t_{j-1}\right) \\
& -\int_{-a / 2}^{a / 2} \prod_{j=1}^{N-1} \mathrm{~d} x_{j} \prod_{j=1}^{N} K_{0}\left(x_{j}, t_{j-1}+\varepsilon ; x_{j-1}, t_{j-1}\right)
\end{aligned}
$$

We can rewrite the propagator in (8) as

$$
K\left(x_{b}, t_{b} ; x_{a}, t_{a}\right)=K_{0}\left(x_{b}, t_{b} ; x_{a}, t_{a}\right)+\delta K\left(x_{b}, t_{b} ; x_{a}, t_{a}\right)
$$

The first term in (17) is the free propagator (see also Ref. [14])

$$
\begin{aligned}
K_{0}\left(x_{b}, t_{b} ; x_{a}, t_{a}\right)= & \int \frac{1}{2}\left(I_{4}+\frac{H_{0}(k)}{E(k)}\right) \mathrm{e}^{\left[\mathrm{i} k\left(x_{b}-x_{a}\right)-\frac{\mathrm{i}}{\hbar} E(k) \varepsilon\right]} \frac{\mathrm{d} k}{2 \pi} \\
& +\int \frac{1}{2}\left(I_{4}-\frac{H_{0}(k)}{E(k)}\right) \mathrm{e}^{\left[\mathrm{i} k\left(x_{b}-x_{a}\right)+\frac{\mathrm{i}}{\hbar} E(k) \varepsilon\right]} \frac{\mathrm{d} k}{2 \pi}
\end{aligned}
$$

which contains the states of positive energy, $E(k)$, in the first term and the states of negative energy, $-E(k)$, in the second term. This propagation of a given spinor both types of states enter the procedure, in general.

For the second term is the propagator due to square well potential, 
called the perturbed propagator $\delta K$, reads

$$
\begin{aligned}
\delta K\left(x_{b}, t_{b} ; x_{a}, t_{a}\right)= & \lim _{N \rightarrow \infty} \frac{1}{(2 \pi)^{N}} \int_{-a / 2}^{a / 2} \prod_{j=1}^{N-1} \mathrm{~d} x_{j} \prod_{j=1}^{N} \mathrm{~d} k_{j} \kappa_{j} \\
& \times \mathrm{e}^{\mathrm{i} k_{j}\left(x_{j}-x_{j-1}\right)}\left[\mathrm{e}^{-\frac{\mathrm{i}}{\hbar} \frac{\varepsilon}{N} V\left(x_{j}\right)}-1\right]
\end{aligned}
$$

To evaluate (19) we rearrange the exponential terms in the form $\operatorname{expi}\left(k_{j}-k_{j+1}\right) x_{j}$, where $j=1,2, \ldots, N-1$. We can then integrate the various exponential term over $x_{j}$ using the identity

$$
\int_{-a / 2}^{a / 2} \mathrm{~d} x \mathrm{e}^{i b x}=\frac{2 \sin [b(a / 2)]}{b}
$$

resulting in the product of sine functions and two plane wave as

$$
\begin{aligned}
& \frac{\sin \left[\left(k_{N-1}-k_{N}\right) \frac{a}{2}\right]}{\left(k_{N-1}-k_{N}\right)} \frac{\sin \left[\left(k_{N-2}-k_{N-1}\right) \frac{a}{2}\right]}{\left(k_{N-2}-k_{N-1}\right)} \ldots \frac{\sin \left[\left(k_{2}-k_{3}\right) \frac{a}{2}\right]}{\left(k_{2}-k_{3}\right)} \\
& \times \frac{\sin \left[\left(k_{1}-k_{2}\right) \frac{a}{2}\right]}{\left(k_{1}-k_{2}\right)} \exp \left[\left(\mathrm{i}_{N} x_{N}-\mathrm{i} k_{1} x_{0}\right)\right]
\end{aligned}
$$

These integrations do not include the potential term becuase we set $V(x)=-V_{0}$ which is just a constant

Then integrating over $k_{j}$ when $j=1, \ldots, N-1$ and setting $x_{0}=x_{a}$, $x_{N}=x_{b}, k_{N}=k$, Equation (19) becomes

$$
\begin{aligned}
\delta K\left(x_{b}, t_{b} ; x_{a}, t_{a}\right)= & \lim _{N \rightarrow \infty} \int\left[I_{4}-\frac{\mathrm{i}}{\hbar}\left(c \hbar \alpha_{x} k+m_{0} c^{2} \beta\right) \frac{\varepsilon}{N}\right]^{N} \\
& \times \exp \left[\mathrm{i} k\left(x_{b}-x_{a}\right)\right]\left[\exp \left(\frac{\mathrm{i}}{\hbar} \varepsilon V_{0}\right)-1\right] \frac{\mathrm{d} k}{2 \pi}
\end{aligned}
$$

The limit as $N \rightarrow \infty$ can now be taken and leads to

$$
\begin{aligned}
\delta K\left(x_{b}, t_{b} ; x_{a}, t_{a}\right)= & \int \exp \left[-\frac{\mathrm{i}}{\hbar}\left(c \hbar \alpha_{x} k+m_{0} c^{2} \beta\right) \varepsilon\right] \\
& \times \exp \left[\mathrm{i} k\left(x_{b}-x_{a}\right)\right]\left[\exp \left(\frac{\mathrm{i}}{\hbar} \varepsilon V_{0}\right)-1\right] \frac{\mathrm{d} k}{2 \pi}
\end{aligned}
$$

By replacing the operator $-\mathrm{i} \hbar \partial / \partial x$ in the free Dirac Hamiltonian $H_{0}$ with $\hbar k$, the free Dirac Hamiltonian in momentum vaiable is $H_{0}(k)=$ $c \hbar \alpha_{x} k+m_{0} c^{2} \beta$. With the aid of the anticommutation relations of the matrices $\alpha_{x}$ and $\beta$, it is easy to show that $H_{0}^{2}(k)=\left[(c \hbar k)^{2}+\right.$ $\left.\left(m_{0} c^{2}\right)^{2}\right] I_{4}=(E(k))^{2}$, where $E(k)$ will denote the positive root as $E(k)=+\left[(c \hbar k)^{2}+m_{0}^{2} c^{4}\right]^{1 / 2}$.

Next, with the aid of the first term of an exponential in (23) we are able to write

$$
\exp \left(-\frac{\mathrm{i}}{\hbar} H_{0} \varepsilon\right)=\cos \left[\frac{1}{\hbar} E(k) \varepsilon\right] I_{4}-\mathrm{i} \sin \left[\frac{1}{\hbar} E(k) \varepsilon\right] \frac{H_{0}(k)}{E(k)}
$$


Equation (24) inserted into (23) gives an expression for the required propagator

$$
\begin{aligned}
\delta K\left(x_{b}, t_{b} ; x_{a}, t_{a}\right)= & \int\left\{\cos \left[\frac{1}{\hbar} E(k) \varepsilon\right] I_{4}-\mathrm{i} \sin \left[\frac{1}{\hbar} E(k) \varepsilon\right] \frac{H_{0}(k)}{E(k)}\right\} \\
& \times \exp \left[\mathrm{i} k\left(x_{b}-x_{a}\right)\right]\left[\exp \left(\frac{\mathrm{i}}{\hbar} \varepsilon V_{0}\right)-1\right] \frac{\mathrm{d} k}{2 \pi}
\end{aligned}
$$

Clearly, (25) satisfies the bounded particles Dirac equation and at the same time as $t_{b} \rightarrow t_{a}$, it goes to $I_{4} \delta\left(x_{b}-x_{a}\right)$. It can therefore propagate any spinor given at $t_{b}=t_{a}$ evolving then via the Dirac equation. The matrix structure of the propagator this is in a simple form since it only depends linearly on the off-diagonal matrix $H_{0}(k)$.

We shall now put (25) into a more transparent form, as far as the energy spectrum is concerned. To this end we just decompose the trigonometric functions into their combinations of exponentials. We have

$$
\begin{aligned}
\delta K\left(x_{b}, t_{b} ; x_{a}, t_{a}\right) & \\
= & {\left[\int \frac{1}{2}\left(I_{4}+\frac{H_{0}(k)}{E(k)}\right) \mathrm{e}^{\left[\mathrm{i} k\left(x_{b}-x_{a}\right)-\frac{\mathrm{i}}{\hbar} E(k) \varepsilon\right]} \frac{\mathrm{d} k}{2 \pi}\right.} \\
& \left.+\int \frac{1}{2}\left(I_{4}-\frac{H_{0}(k)}{E(k)}\right) \mathrm{e}^{\left[\mathrm{i} k\left(x_{b}-x_{a}\right)+\frac{\mathrm{i}}{\hbar} E(k) \varepsilon\right]} \frac{\mathrm{d} k}{2 \pi}\right]\left[\mathrm{e}^{\frac{\mathrm{i}}{\hbar} \varepsilon V_{0}}-1\right]
\end{aligned}
$$

By substituting (14) and (26) into (17), we obtain the propagator for Dirac particles confined in an one-dimensional finite square well potential as

$$
\begin{aligned}
& K\left(x_{b}, t_{b} ; x_{a}, t_{a}\right) \\
& =\int \frac{1}{2}\left(I_{4}+\frac{H_{0}(k)}{E(k)}\right) \mathrm{e}^{\left[\mathrm{i} k\left(x_{b}-x_{a}\right)-\frac{\mathrm{i}}{\hbar}\left[E(k)-V_{0}\right] \varepsilon\right]} \frac{\mathrm{d} k}{2 \pi} \\
& +\int \frac{1}{2}\left(I_{4}-\frac{H_{0}(k)}{E(k)}\right) \mathrm{e}^{\left[\mathrm{i} k\left(x_{b}-x_{a}\right)-\frac{\mathrm{i}}{\hbar}\left[-E(k)-V_{0}\right] \varepsilon\right]} \frac{\mathrm{d} k}{2 \pi}
\end{aligned}
$$

In (27), we say that the propagator for bounded particles with potential $-V_{0}$ are obtained from the free propagator by the subsitution $\pm E \rightarrow \pm E-V_{0}$. In this case no spin-flip occurs at the border of the well. The allowed energies can take all values from $-\infty$ up to $\infty$, so that we can describe particles as well as antiparticles.

By performing the integrations over $k$ and making use of the functions $\varphi\left(x^{0}, \lambda\right)$

$$
\varphi\left(x^{0}, \lambda\right)= \begin{cases}\frac{1}{2 \mathrm{i}} N_{0}(m \sqrt{\lambda})-\frac{1}{2} E\left(x^{0}\right) J_{0}(m \sqrt{\lambda}) & \text { for } \lambda>0 \\ \frac{\mathrm{i}}{\pi} K_{0}(m \sqrt{-\lambda}) & \text { for } \lambda<0\end{cases}
$$

with $m=m_{0} c / \hbar, x^{0}=c \varepsilon, \varepsilon=t_{b}-t_{a}, r=\left|x_{b}-x_{a}\right|, \eta=\left(1-V_{0} / E(k)\right)$ and $\lambda=\left(\eta x^{0}\right)^{2}-r^{2}$, defined in Bogoliubov and Shirkov [25]. The Dirac propagator for Dirac particles confined in an one-dimensional finite square well potential is thus 


$$
\begin{aligned}
& K\left(x_{b}, t_{b} ; x_{a}, t_{a}\right) \\
& =\frac{1}{2}\left[I_{4} \frac{\partial}{\partial x^{0}}-\alpha_{x} \frac{\partial}{\partial r}-\mathrm{i} m \beta\right]\left[\frac{1}{4 \pi} \frac{\partial}{\partial r}\left\{\varphi\left(x^{0}, \lambda\right)+\varphi^{*}\left(x^{0}, \lambda\right)\right\}\right] \\
& =\frac{1}{2}\left[I_{4} \frac{\partial}{\partial x^{0}}-\alpha_{x} \frac{\partial}{\partial r}-\mathrm{i} m \beta\right] \\
& {\left[\frac{1}{2 \pi} E\left(x^{0}\right) \delta(\lambda)-\frac{m}{4 \pi \sqrt{-\lambda}} \theta(\lambda) E\left(x^{0}\right) J_{1}(m \sqrt{\lambda})\right]}
\end{aligned}
$$

\section{Conclusion}

This paper applied the path-integral formalism to solve the Dirac equation of Dirac particles confined in a one-dimensional finite square well potential of depth $V_{0} \leq 0$ and width $a$. The Dirac propagator which is obtained by evaluating an appropriate path integral, directly constructed from the Dirac equation, is expressed in terms of standard special functions as in Equation (29). We note that the limit of this technique for evaluating the propagator is only valid for a square well potential, which is a piecewise constant potential. For a more general potential new technique is needed. One possible direction is to write down the path integral for the special wave equation associated with the Dirac equation instead of writing down the path integral directly [14].

\section{Acknowledgements}

The work of Phonphimon Kongkhuntod and Nattapong Yongram has been supported by the Faculty of Science Funding. We would like to thank Asst. Prof. Dr. Sikarin Yoo-Kong for discussions and guidance. We would like to thank Dr.Watee Srinin for discussions, guidance and manuscript preparation. We also would like to thank Sujin Wangsuya for discussions and comments. Finally, we would like to acknowledge with thanks the Department of Physics, Naresuan University, Phitsanulok, Thailand, for its kind hospitality.

\section{Conflicts of Interest}

The authors declare no conflicts of interest regarding the publication of this paper.

\section{References}

[1] Ciftja, O. and Johnston, B. (2019) European Journal of Physics, 40, Article ID: 045402. https://doi.org/10.1088/1361-6404/ab1a61

[2] Ewa, I.I., Howusu, S.X.K. and Lumbi, L.W. (2019) Physical Science International Journal, 22, 1-9. https://doi.org/10.9734/psij/2019/v22i330134

[3] Al-Ani, L.A. and Abid, R.K. (2019) Al-Nahrain Journal of Science, 22, 52-58. https://doi.org/10.22401/ANJS.22.4.07 
[4] Ewa, I.I., Lumbi, L.W. and Howusu, S.X.K. (2018) International Journal of Theoretical and Mathematical Physics, 8, 28-31.

[5] Roberts, K. and Valluri, S.R. (2017) Canadian Journal of Physic$s$, 95, 105-110. https://doi.org/10.1139/cjp-2016-0602

[6] Coulter, B.L. and Adler, C.G. (1971) Journal of American Mathematical Society, 39, 305-309.

[7] Gumbs, G. and Kiang, D. (1986) Journal of American Mathematical Society, 54, 462-463.

[8] Greiner, W. and Bromley, D.A. (2000) Relativistic Quantum Mechanics. 3rd Edition, Springer, Berlin. https://doi.org/10.1007/978-3-662-04275-5

[9] Nevels, R.D., Wu, Z. and Huang, C. (1993) Physical Review A, 48, 3445-3451. https://doi.org/10.1103/PhysRevA.48.3445

[10] Janke, W. and Kleinert, H. (1979) Lettere al Nuovo Cimento, 25, 297-300. https://doi.org/10.1007/BF02776259

[11] Goodman, M. (1981) Journal of American Mathematical Society, 49, 843-847.

[12] Barut, A.O. and Duru, I.H. (1988) Physical Review A, 38, 59065909. https://doi.org/10.1103/PhysRevA.38.5906

[13] Riazanov, G.V. (1958) Journal of Experimental and Theoretical Physics, 6, 1107-1113.

[14] Papadopoulos, G.J. and Devreese, J.T. (1976) Physical Review D, 13, 2227-2234. https://doi.org/10.1103/PhysRevD.13.2227

[15] Feynman, R.P. (1942) The Principle of Least Action in Quantum Mechanics. Ph.D. Thesis, Princeton University, Princeton.

[16] Feynman, R.P. Brown, L.M. and Dirac, P.A.M. (2005) Feynmans Thesis: A New Approach to Quantum Theory. World Scientific, Hackensack, NJ.

[17] Feynman, R.P. (1948) Review Modern Physics, 20, 367-387. https://doi.org/10.1103/RevModPhys.20.367

[18] Feynman, R.P. and Hibbs, A.R. (1965) Quantum Mechanics and Path Integrals. McGraw-Hill College, New York.

[19] Gaveau, B. and Schulman, L.S. (2000) Annals of Physics, 284, 1-9. https://doi.org/10.1006/aphy.1999.5993

[20] Rosen, G. (1983) Physical Review A, 28, 1139-1140. https://doi.org/10.1103/PhysRevA.28.1139

[21] Naimark, M.A. and Stern, A.I. (1982) Theory of Group Representation. Springer, Berlin, Heidelberg.

[22] Suzuki, T. (1977) Communications in Mathematical Physics, 57, 193-200. https://doi.org/10.1007/BF01614161

[23] Wilcox, R.M. (1967) Journal of Mathematical Physics, 8, 962982. 
[24] Grosche, C. and Steiner, F. (1997) Handbook of Feyman Path Integrals. Springer-Verlag, Berlin.

[25] Bogoliubov, N.N. and Shirkov, D.V. (1959) Introduction to Quantized Fields. Wiley, New York, 147-150. 